\section{Vol. 32, Issue 8-9, September 2009}

\section{Editorials}

462 The Reawakening of Bendamustine - Also in Breast Cancer?

von Minckwitz, G.; Linder, M.; Loibl, S. (Neu-Isenburg)

464 Adjuvant Anthracyclines and Taxanes: Quantifying the Costs of Therapeutic Advances in Breast Cancer Morris, P.G.; Bach, P.B. (New York, NY)

Original Articles

468 Doxorubicin and Paclitaxel versus Fluorouracil, Doxorubicin and Cyclophosphamide as First-Line Therapy for Women with Advanced Breast Cancer: Long-Term Analysis of the Previously Published Trial

Jassem, J. (Gdansk); Pienkowski, T. (Warsaw); Pluzanska, A. (Lodz); Jelic, S. (Belgrade); Gorbunova, V. (Moscow); Berzins, J. (Riga); Nagykalnai, T. (Budapest); Biganzoli, L. (Prato); Aloe, A.; Astier, L.; Munier, S. (Braine-l'Alleud)

473 Cost Analysis Comparing an Anthracycline/Docetaxel Regimen to CMF in Patients with Early Stage Breast Cancer

Braun, M. (München/Bonn); Jacobs, V.R.; Wagenpfeil, S.;

Sattler, D.; Harbeck, N. (München); Nitz, U. (Mönchengladbach); Bernard, R. (München); Kuhn, W. (München/Bonn); Ihbe-Heffinger, A. (München)

482 Tolerability of Dose Escalation of Ibandronate in Patients with Multiple Myeloma and End-Stage Renal Disease: A Case Series Henrich, D.M. (Saarlouis/Ludwigshafen); Hoffmann, M.; Uppenkamp; M., Bergner, R. (Ludwigshafen)

488 Phase II Study with 3rd- or 4th-Line Bendamustine (Flat Dose) Therapy in Patients with Metastatic Breast Cancer

Steinbild, S.; Frost, A.; Häring, B.; Unger, C.; Mross, K. (Freiburg)

493 Impact of Treatment Guidelines and Implementation of a Quality Assurance Program on Quality of Care in Endometrial Cancer du Bois, A.; Strutas, D.; Buhrmann, C.; Traut, A.; Ewald-Riegler, N.; Kommoss, S.; Gomez, R.; Harter, P. (Wiesbaden)
Band 32, Heft 8-9, September 2009

Editorial

462 Das Wiedererwachen von Bendamustin - auch beim Mammakarzinom?

von Minckwitz, G.; Linder, M.; Loibl, S. (Neu-Isenburg)

464 Adjuvante Anthrazykline und Taxane: Bewertung der Kosten von therapeutischen Fortschritten in der Brustkrebstherapie

Morris, P.G.; Bach, P.B. (New York, NY)

Originalarbeiten

468 Doxorubicin und Paclitaxel versus Fluorouracil, Doxorubicin und Cyclophosphamid als FirstLine-Therapie für Frauen mit fortgeschrittenem Mammakarzinom: Langzeitanalyse der Studiendaten

Jassem, J. (Gdansk); Pienkowski, T. (Warsaw); Pluzanska, A. (Lodz); Jelic, S. (Belgrade); Gorbunova, V. (Moscow); Berzins, J. (Riga); Nagykalnai, T. (Budapest); Biganzoli, L. (Prato); Aloe, A.; Astier, L.; Munier, S. (Braine-l'Alleud)

473 Kosten-Analyse von sequenziellem Docetaxel im Vergleich zu CMF bei Patientinnen mit primärem Mammakarzinom Braun, M. (München/Bonn); Jacobs, V.R.; Wagenpfeil, S.; Sattler, D.; Harbeck, N. (München); Nitz, U. (Mönchengladbach); Bernard, R. (München); Kuhn, W. (München/Bonn); Ihbe-Heffinger, A. (München)

482 Verträglichkeit der Dosiseskalation von Ibandronat bei Patienten mit multiplem Myelom und terminaler Niereninsuffizienz: Eine Fallserie Henrich, D.M. (Saarlouis/Ludwigshafen); Hoffmann, M.; Uppenkamp; M., Bergner, R. (Ludwigshafen)

488 Phase-II-Studie zur Therapie mit Bendamustin beim metastasierten Mammakarzinom Steinbild, S.; Frost, A.; Häring, B.; Unger, C.; Mross, K. (Freiburg)

493 Der Einfluss von klinikinternen Standards und Qualitätssicherungsmaßnahmen auf die Therapiequalität beim Endometriumkarzinom du Bois, A.; Strutas, D.; Buhrmann, C.; Traut, A.; Ewald-Riegler, N.; Kommoss, S.; Gomez, R.; Harter, P. (Wiesbaden)

\section{KARGER}

Fax +49761 4520714

Information@Karger.de

www.karger.com 


\section{Vol. 32, Issue 8-9, September 2009}

Clinical Cases

499 Primitive Neuroectodermal Tumor of the Pelvis in an Elderly Patient

Saada, E.; Thariat, J.; Follana, P.; Birtwisle-Peyrottes, I.;

Haudebourg, J.; Trojani, C.; Bacque, P.; Thyss, A. (Nice)

503 Treatment of a Malignant Peripheral Nerve Sheath Tumor

Lin, C.-T.; Huang, T.-W.; Nieh, S.; Lee, S.-C. (Taipei)

506 Lymphoplasmacytic Sclerosing Pancreatitis with Obstructive Jaundice: A Case Report and Review of the Literature

Wang, G.; Zhu, H.; Yuan, C.-X. (Harbin); Gao, Y. (Gothenburg); Li, J.; Xue, D.-B.; Sun, B. (Harbin)

509 Radiofrequency Ablation as a Palliative Treatment of Primary Liver Sarcoma in an Inoperable Patient with Hepatitis C Virus-Related Cirrhosis Chen, J.-H.; Chang, P.-Y.; Ho, C.-L.; Chen, P.-J. (Taipei)

513 Precursor T-Lymphoblastic Lymphoma as a Secondary Malignancy in a Young Patient after Successful Treatment of Acute Promyelocytic Leukemia

Szotkowski, T.; Jarosova, M.; Faber, E.; Hubacek, J.; Hlusi, A.; Papajik, T.; Pikalova, Z.; Kucerova, L.; Holzerova, M.; Budikova, M.; Buriankova, E.; Plachy, R.; Potomkova, J.; Klusova, N.; Szotkowska, R.; Indrak, K. (Olomouc)

Review Article

517 Paraneoplastic Humoral Mediated Hypercalcemia Induced by Parathyroid Hormone-Related Protein in Gynecologic Malignancies:

A Systematic Review Savvari, P.; Peitsidis, P.; Alevizaki, M.; Dimopoulos, M.-A.; Antsaklis, A.; Papadimitriou, C.A. (Athens)

526 PharmaForum

532 PharmaNews / PharmaTicker

536 Meetings and Conferences

538 Guidelines for Authors

481 Imprint

Forthcoming papers are listed on page 540.

\section{Band 32, Heft 8-9, September 2009}

Kasuistiken

499 Primitiver neuroektodermaler Tumor der Beckenregion bei einem älteren Patienten

Saada, E.; Thariat, J.; Follana, P.; Birtwisle-Peyrottes, I.;

Haudebourg, J.; Trojani, C.; Bacque, P.; Thyss, A. (Nice)

503 Behandlung eines malignen peripheren

Nervenscheidentumors

Lin, C.-T.; Huang, T.-W.; Nieh, S.; Lee, S.-C. (Taipei)

506 Lymphoplasmazytäre sklerosierende Pankreatitis mit obstruktivem Ikterus: Fallbericht und

Literaturübersicht

Wang, G.; Zhu, H.; Yuan, C.-X. (Harbin); Gao, Y. (Gothenburg); Li, J.; Xue, D.-B.; Sun, B. (Harbin)

509 Radiofrequenzablation als Palliativbehandlung eines primären Lebersarkoms bei einem inoperablen Patienten mit Hepatitis-C-Virus-bedingter Zirrhose Chen, J.-H.; Chang, P.-Y.; Ho, C.-L.; Chen, P.-J. (Taipei)

513 Vorläufer-T-lymphoblastisches Lymphom als Sekundärmalignom bei einer jungen Patientin nach erfolgreicher Behandlung einer akuten promyeloischen Leukämie

Szotkowski, T.; Jarosova, M.; Faber, E.; Hubacek, J.; Hlusi, A.; Papajik, T.; Pikalova, Z.; Kucerova, L.; Holzerova, M.; Budikova, M.; Buriankova, E.; Plachy, R.; Potomkova, J.; Klusova, N.; Szotkowska, R.; Indrak, K. (Olomouc)

Übersichtsarbeit

517 Paraneoplastische, humoral vermittelte Hyperkalzämie, die bei gynäkologischen Malignomen durch das parathormonverwandte Protein induziert wird: Eine systematische Übersicht Savvari, P.; Peitsidis, P.; Alevizaki, M.; Dimopoulos, M.-A.; Antsaklis, A.; Papadimitriou, C.A. (Athens)

526 PharmaForum

532 PharmaNews / PharmaTicker

536 Tagungen und Kongresse

539 Hinweise für Autoren

481 Impressum

Einen Ausblick auf den Inhalt der kommenden Hefte finden Sie auf Seite 540.

\section{KARGER}

Fax +497614520714

Information@Karger.de

www.karger.com

\section{(C) 2009 S. Karger GmbH, Freiburg}

Artikel (Volltext) und Inhaltsverzeichnisse

sowie das vorläufige Inhaltsverzeichnis des nächsten Heftes:

www.karger.com/onk_bk.htm 